OPEN ACCESS

Edited by:

Bruno Laugel,

Cardiff University School of Medicine,

Reviewed by:

Koji Yasutomo,

University of Tokushima, Japan

Linda Wooldridge,

University of Bristol, UK

*Correspondence:

Ludvig A. Munthe,

Department of Immunology, Centre

for Immune Regulation, Institute of Clinical Medicine, University of Oslo,

Postboks 4953 Nydalen,

Oslo 0424, Norway

I.a.munthe@medisin.uio.no

Specialty section:

This article was submitted to T Cell Biology, a section of the journal

Frontiers in Immunology

Received: 19 May 2015

Accepted: 13 July 2015

Published: 28 July 2015

Citation:

Aas-Hanssen K, Thompson KM,

Bogen B and Munthe LA (2015)

Systemic lupus erythematosus:

molecular mimicry between

anti-dsDNA CDR3 idiotype, microbial

and self peptides - as antigens

for Th cells.

Front. Immunol. 6:382.

doi: 10.3389/fimmu.2015.00382

\section{Systemic lupus erythematosus: molecular mimicry between anti-dsDNA CDR3 idiotype, microbial and self peptides - as antigens for Th cells}

\author{
Kristin Aas-Hanssen ${ }^{1}$, Keith M. Thompson ${ }^{1}$, Bjarne Bogen ${ }^{1,2}$ and Ludvig A. Munthe ${ }^{1 *}$ \\ ${ }^{1}$ Department of Immunology, Centre for Immune Regulation, Institute of Clinical Medicine, University of Oslo, Oslo, Norway, \\ ${ }^{2}$ KG Jebsen Centre for Influenza Vaccine Research, Institute of Clinical Medicine, University of Oslo, Oslo, Norway
}

Systemic lupus erythematosus (SLE) is marked by a T helper (Th) cell-dependent B cell hyperresponsiveness, with frequent germinal center reactions, and gammaglobulinemia. A feature of SLE is the finding of IgG autoantibodies specific for dsDNA. The specificity of the Th cells that drive the expansion of anti-dsDNA B cells is unresolved. However, antimicrobial, anti-histone, and anti-idiotype Th cell responses have been hypothesized to play a role. It has been entirely unclear if these seemingly disparate Th cell responses and hypotheses could be related or unified. Here, we describe that $\mathrm{H}$ chain CDR3 idiotypes from $\operatorname{lgG}+$ B cells of lupus mice have sequence similarities with both microbial and self peptides. Matched sequences were more frequent within the mutated CDR3 repertoire and when sequences were derived from lupus mice with expanded anti-dsDNA B cells. Analyses of histone sequences showed that particular histone peptides were similar to VDJ junctions. Moreover, lupus mice had Th cell responses toward histone peptides similar to anti-dsDNA CDR3 sequences. The results suggest that Th cells in lupus may have multiple cross-reactive specificities linked to the IgVH CDR3 Id-peptide sequences as well as similar DNA-associated protein motifs.

Keywords: molecular mimicry, systemic lupus erythematosus, idiotypes, B cells, Th cells, B cell receptor, complementarity determining region 3 , antigen presentation

\section{Introduction}

The initiation of autoimmune responses is associated with infection and the development of a gradually evolving $\mathrm{T}$ cell and $\mathrm{B}$ cell autoreactive response toward self-proteins. Molecular mimicry is the concept that similarities of microbial peptides to self-peptides can allow expansion of microbial specific $\mathrm{T}$ cells that can cross react to similar self-peptides $(1,2)$. Molecular mimicry has been suggested to play a part in diverse autoimmune diseases such as multiple sclerosis, rheumatoid arthritis, diabetes, stromal keratitis, myocarditis, and inflammatory bowel disease $(1,2)$. It was originally believed that TCR binding to peptide:MHC (pMHC) was dependent upon stringent requirements for amino acid (aa) identity of the $\mathrm{T}$ cell contact residues. It is increasingly clear that degeneracy in both the TCR $(3,4)$ and MHC (5) peptide-binding motifs as well as interchangeable aa with similar properties in the TCR-exposed aa motifs $(4,6,7)$ reduce this sequence-specific requirement (1). 
Anti-dsDNA B cells and high titers of nephrotoxic anti-dsDNA autoantibodies are hallmarks of systemic lupus erythematosus (SLE) (8). Molecular mimicry has not been studied extensively in SLE, but a role for Th cells is well-established; it is clear that the expansion of autoreactive $B$ cell requires pathogenic Th cells (8-11). However, the antigen specificity of the Th cells has been unclear. Candidate antigens include peptides derived from nucleic acid-associated proteins such as small nuclear riboprotein (12), histones (13), or similar proteins derived from bacteria or viruses (14). Hence, anti-DNA/RNA B cells could bind and endocytose nucleic acids as well as DNA- or RNA-associated proteins. Th cells that are specific for peptides derived from such self proteins or similar peptides derived from pathogens could thereafter collaborate with the B cells.

Another candidate for Th cell antigen is variable regions of antibody, i.e., $\mathrm{V}$ region idiotypes, Id. Th cells can have Id:MHCspecific TCR (15-33). It has been demonstrated that anti-dsDNA B cells can present Id:MHC class II to Id-specific Th cells, undergo the germinal center reaction, differentiate, secrete autoantibodies, and cause vasculitis and nephritis (28, 30, 31, 33). Moreover, Id-specific Th cell responses increased with disease severity in lupus mice, and disease was aggravated by injection of Id-peptide analogs (22).

We here hypothesize that these disparate suggestions for Th cell antigen in lupus are examples drawn from a network of peptide mimics. RNA/DNA-associated proteins and anti-dsDNA antibodies both have positively charged (cationic) nucleotidebinding motifs. Thus, Th cells that are specific for cationic microbial peptides may cross react with B cells presenting cationic self peptides or Id peptides from anti-DNA BCR. If so, Th cells could support B cells that present such peptide mimics thereby allowing autoantibody secretion.

We therefore aimed to analyze if the seemingly dissimilar Th cell specificities for cationic CDR3 idiotypes and cationic DNAbinding peptides could constitute networks of molecular mimics for Th cells in SLE. We chose to compare heavy chain junctions (IgVH CDR3 peptides) from mice with lupus with proteomes from microbes and mouse. The CDR3 of the heavy chain has the highest potential for novel peptides, both in terms of $\mathrm{N}$ region diversity (V-N1-D-N2-J) as well as the option of alternate reading frames of the $\mathrm{D}$ gene segment, and as a site for mutations.

We first compared IgVH CDR3 idiotypes from lupus mice with bacterial proteomes, thereafter viral proteins and self proteins. Using bioinformatics analyses, we found a surprisingly high rate of matches between CDR3 sequences and microbial proteomes as well as with self proteins, including histones. We also found that mice suffering from Id-driven lupus (with high levels of anti-dsDNA responses) developed Th cell responses toward antidsDNA mAbs with CDR3 sequences that resembled histones, suggesting epitope spreading involving cationic peptide mimics including idiotypes and self proteins.

\section{Materials and Methods}

\section{Mice}

Mice were transgenic for both the $\lambda 2^{315} \mathrm{Ig} \mathrm{L}$-chain derived from the MOPC315 myeloma, as well as a TCR $\alpha \beta$ transgene specific for the $\operatorname{Id}\left(\lambda 2^{315}\right)$-peptide presented on I-E ${ }^{\mathrm{d}}$ MHC class II molecules $(30,31)$. These double transgenic mice are called DTG mice herein. The Norwegian Animal Research Authority approved the experiments.

\section{IgVH Sequence Processing and Analysis}

The following control data sets were downloaded from $\mathrm{NCBI}^{1}$. 1) control $\mathrm{VH}$ sequences from the $\mathrm{BALB} / \mathrm{c}$, retrieved with the search term "V region immunoglobulin heavy chain BALB," 2) Sequences derived from splenic $\mathrm{L} 2-\mathrm{TG}$ mice $\mathrm{IgG}^{+}$B cells as deposited (34), 3) neonatal liver B cell $\operatorname{IgVH}$ sequences as deposited (35).

Sequences were analyzed with the IMGT/HighV-QUEST version: 1.1.2 or IMGT/HighV-QUEST version: 3.2 .30 tools $^{2}$ and compared to the IMGT/V-QUEST reference directory release: 201310-4 (14 March 2013) (36). IgVH region family identification and clonality analysis were performed on the statistics module of IMGT/HighV-QUEST. Statistics were reported only for unique sequences (36). Translated aa sequences were further analyzed in Excel (Microsoft), aa in individual positions were counted with the "countif" function.

\section{Search for Similarities Between CDR3 and Microbial, Self and Viral Proteomes}

Only CDR3 sequences from 11-16 aa length were included in the analysis, sequences with CDR3 length $11,12,13$, and 14 included FR3 aa $(4,3,2$, and 1 aa, respectively) so that 15 mers (or 16mers) were used. CDR3 sequences were analyzed on the Basic Local Alignment Search Tool (BLAST) server ${ }^{3}$ with the Blastp suite (protein-protein BLAST) and search parameters were adjusted to search for a short input sequence. CDR3 peptides were compared to 1) the microbial genomes (BLOSUM62 matrix), constituting mostly bacterial species (see list in the Supplementary Material); 2) the mouse proteome utilizing the mouse Refseq genome; 3) CDR3 sequences were also compared to non-redundant databases (GenBank CDS translations $+\mathrm{PDB}+$ SwissProt $+\mathrm{PIR}+\mathrm{PRF}$ excluding environmental samples from WGS projects). Resulting sequence matches were manually screened for sequences of microorganism origin; 4) sequence analyses were performed against viral databases at the Viral Bioinformatics Resource Center ${ }^{4}$. Figures 1 and 2 show analysis of sequences compared to the Herpesviridae protein database $(11,887$ sequences; $5,156,626$ total letters, database posted June 14, 2012).

Alignments and matches were performed by registering only contiguous, i.e. non-gap'-ed sequences. aa equivalence was scored according to BLAST algorithms.

To analyze similarities between previously published VH CDR3 sequences and histones, we made artificial sequence files where variously truncated DNA-binding motifs from histones $(\mathrm{H} 1, \mathrm{H} 2 \mathrm{~A}$, $\mathrm{H} 4)$ were inserted into sequences starting with the aa CAR and terminating with four to six aa from FR4. Artificial sequence files

\footnotetext{
${ }^{1}$ http://www.ncbi.nlm.nih.gov/nuccore/

${ }^{2}$ http://www.imgt.org/

${ }^{3}$ http://blast.ncbi.nlm.nih.gov/Blast.cgi

${ }^{4}$ http://athena.bioc.uvic.ca/virology-ca-tools/blast/
} 


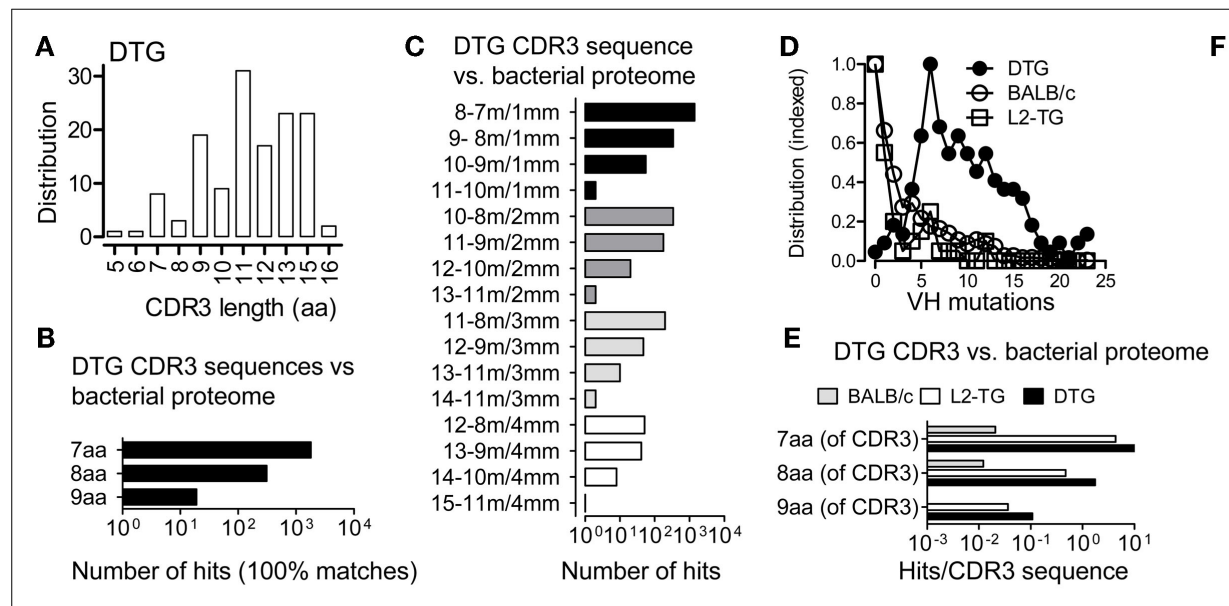

C DTG CDR3 sequence vs. bacterial proteome $8-7 \mathrm{~m} / 1 \mathrm{~mm}$ $9-8 \mathrm{~m} / 1 \mathrm{~mm}$ $0-9 \mathrm{~m} / 1 \mathrm{~mm}$ $1-10 \mathrm{~m} / 1 \mathrm{~mm}$ $11 \mathrm{~m} / 3 \mathrm{~mm}$ Number of hits

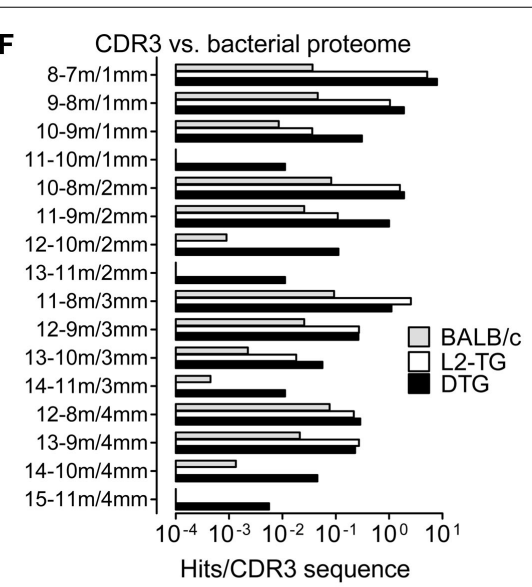

G CDR3 vs. herpesviridae-proteome

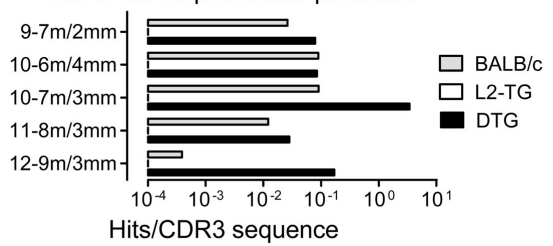

H

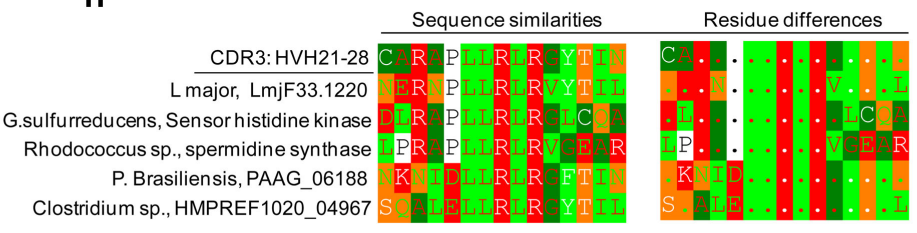

Key: Amino acid properties
FIGURE 1 | Analysis of similarities between CDR3 sequences and the microbial proteome. (A) IgVH CDR3 length distribution of sequences from DTG mice with end stage lupus. (B,C,E-H), Results from Blastp analysis of 15mer CDR3 peptides vs. microbial proteomes and G, Herpesviridae proteome. (B) DTG IgG IgVH CDR3 peptides vs. microbial proteome: Numbers of hits with $100 \%$ matches with contiguous microbial sequences are shown (i.e. alignments without gaps). (C) DTG IgG IgVH CDR3 vs. microbial proteome: Number of hits in the indicated categories of length with matched/mismatched aa. For example: a 12 aa stretch that includes 10 matches $(\mathrm{m})$ and 2 mismatches $(\mathrm{mm})$ is labeled "12-10 $\mathrm{m} / 2 \mathrm{~mm}$."

(D) Mutations per lgVH sequence (not including CDR3) in the three sequence data sets: BALB/c, DTG IgG, L2-TG IgG (see Materials and Methods). The highest frequency of mutations for each data set is normalized to 1 on the $Y$ axis. (E) Exact matches normalized to the number of input sequences in DTG vs. L2-TG IgG data sets. (F) Hits with matches/mismatches [as denoted in (C)] normalized to the number of input sequences in DTG, L2-TG, and BALB/C data sets. (G) Hits with matches/mismatches as in (F), compared to Herpesviridae proteome, normalized to the number of input sequences. (H) Example of one DTG anti-dsDNA IgVH CDR3 sequence and similar microbial sequences. An additional hit from outside the microbial databases is also shown, from the Leishmania major protozoa. Sequences similarities between IgVH CDR3 and proteins are shown in the left panels. Amino acids are color coded according to charge (Negative: D, E; positive: H, K, R), or the chemical properties of side chains (i.e. amide: N, Q; alcohol: $S, T$; aliphatic: L, I, V; aromatic: F, Y, W; small size: A, G; sulfur atom: M, C; or other: P), see key for color code. In the right panels, differences in the sequences are marked by aa symbols when such aa do not belong to the same chemical group as the most frequent aa at the corresponding position of the comparison set. were screened for matches with Ig sequences. In the Blast analyses, only contiguous (ungapped) matches were included. Sequence visualization and generation of similarity and difference plots were done with the GeneDoc program ${ }^{5}$.

\section{Th Cell Culture and In vitro Assays}

Th cells $\left(5 \times 10^{4} /\right.$ well $)$ from lymph nodes of DTG mice, $\mathrm{Id}^{+}$ single transgenic mice (L2-TG) and BALB/c were mixed with 2000 Rad-irradiated BALB/c splenocytes $\left(5 \times 10^{5} /\right.$ well $)$ and $16 / 17$ mer peptides derived from histones as indicated (see below), $\left[{ }^{3} \mathrm{H}\right] \mathrm{TdR}$ was added on day 3 , proliferation was measured as counts on a TopCount NXT Scintillation Counter (PerkinElmer) on day 6.

Th cell lines from lymph nodes of DTG mice, L2-TG mice and $\mathrm{BALB} / \mathrm{c}$ were stimulated by irradiated $\mathrm{BALB} / \mathrm{c}$ splenocytes and indicated peptides, were restimulated in 10 day cycles, IL-2 was

\footnotetext{
${ }^{5}$ http://www.nrbsc.org/gfx/genedoc
}

first provided on first re-stimulation. Th cell lines from DTG mice were negative for the clonotype specific mAb GB113 $(30,31)$ that stains the transgenic Id-specific TCR and did not respond to Id $\left(\lambda 2^{315}\right)$-peptide.

DNA-binding histone sequences were identified, peptides were synthesized by Think Peptides. Histone H2A superfamily: called HisH2A-epitope 1 (ep1), ${ }^{28}$ VGRVHRLLRKGNYAERV ${ }^{44}$ (17mer), HisH2A-ep2: ${ }^{66}$ LAGNAARDNKKTRIIPR ${ }^{82}$ (17mer). Histone H4 (HisH4-ep1): ${ }^{35}$ IRRLARRGGVKRISGL ${ }^{50}$ (16mer). HisH4-ep2: ${ }^{70}$ AVTYTEHAKRKTVTAM ${ }^{85}$ (16mer), histone H1 family, HisH1: ${ }^{75} \mathrm{KNNSRIKLGLKSLVSK}^{90}$ (16mer).

\section{Results}

\section{CDR3 Sequences from Anti-DNA B Cells from Lupus Mice Show Multiple Similarities with Microbial Sequences}

We have previously described $\mathrm{IgVH}$ sequences from lupus prone mice suffering from Id-driven lupus (33). Utilizing a data set of 176 
A CDR3 sequence vs. bacterial proteome

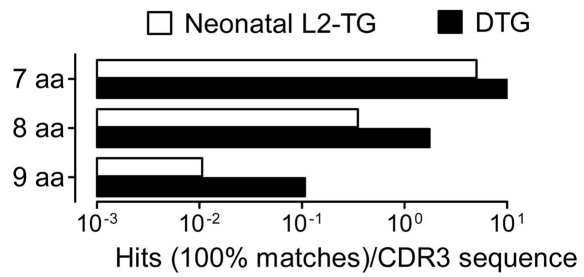

B CDR3 vs. bacterial proteome

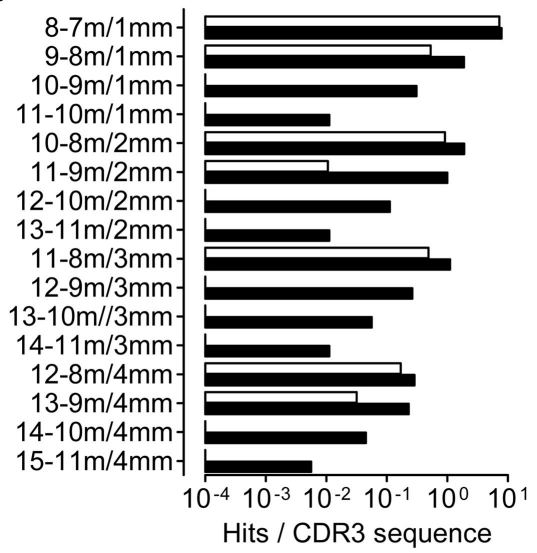

C CDR3 vs. Herpesviridae-proteome

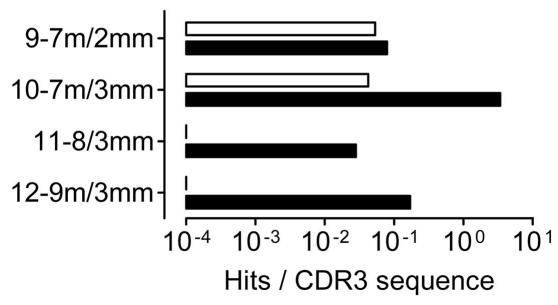

FIGURE 2 | Analysis of CDR3 from IgVH of neonatal sequences, role of somatic mutations. We compared lgVH CDR3/microorganism matches in two data sets derived from the highly mutated DTG sequences and neonatal sequences from L2-TG mice. (A) Exact matches per sequence are shown in contiguous microbial sequences. (B) Hits matches/mismatches in the

microbial proteome are shown (aa length-matches/mismatches, denoted as in Figure 1). (C) Matches including mismatches compared to Herpesviridae proteome, hits normalized to the number of input sequences are shown.

sequences with average of 11 IgVH CDR3 aa (Figure 1A), we compared CDR3 sequences with microbial sequences utilizing Blastp (microbial sequences include non-redundant data from prokaryotic genome sequencing projects, but not viruses or eukaryotic pathogens such as protozoa and fungi), see Materials and Methods. Because $\mathrm{T}$ cells recognize linear epitopes, only contiguous (i.e. non-gap'-ed) matches were analyzed. With this approach, we found frequent hits as 7,8 , or 9 contiguous matched aa could be found in the microbial proteomes (Figure 1B). Moreover, when analyzing sequences in terms of matches including mismatches, further hits were found (Figure 1C), for example the DTG IgVH CDR3 sequences data set had 55 hits in the category 10 contiguous aa with 9 matches $(\mathrm{m}), 1$ mismatch $(\mathrm{mm})$, denoted $10-9 \mathrm{~m} / 1 \mathrm{~mm}$ in the figures.

\section{CDR3 Sequences from B Cells with a High Number of VH Mutations are More Likely to Match Microbial Sequences}

We proceeded to compare the IgVH sequences from DTG lupus mice with sequences derived from $\operatorname{IgG}^{+} \mathrm{B}$ cells from single $\lambda 2^{315}$ transgenic (L2-TG) mice (34). These mice are healthy and have $B$ cells expressing the same $\lambda 2^{315}$ transgene as the current DTG lupus mice. As another unbiased control we used $>2000 \mathrm{BALB} / \mathrm{c}$ sequences (all, deposited and annotated BALB/c sequences, see Materials and Methods). The DTG mice have a high mutation rate in the $\mathrm{VH}$ gene segment compared to the other two data sets, but both L2-TG and BALB/c included mutated sequences (Figure 1D).

Continuing the analysis, we blasted each data set toward the microbial proteome and normalized the rate of hits to the number of analyzed input sequences in each of the three groups. We found that IgVH CDR3 from DTG lupus mice with the highest rate of $\mathrm{VH}$ gene mutations had a 1.4-3.7 increased frequency of exact matches (DTG vs. L2-TG, Figure 1E). A similar tendency was seen in sequences with few mismatched aa (Figure 1F), for example the category with 10 contiguous aa consisting of 9 matches and 1 mismatch $(10-9 \mathrm{~m} / 1 \mathrm{~mm})$, had 0.3 hits per $\mathrm{IgVH}$ CDR3 sequence from the DTG data set, 0.04 per IgVH CDR3 in the sequences from the L2-TG mice and 0.009 per IgVH CDR3 in the sequences from the BALB/c mice. L2-TG IgG sequences had a higher hit rate than $\mathrm{BALB} / \mathrm{c}$ sequences, a finding that may relate to a higher level of marginal zone $B$ cells in the L2-TG mice (34).

To extend the analysis to viral proteomes, we compared the IgVH CDR3 data sequence data sets with the proteome from Herpesviridae, a large family of DNA viruses (see Materials and Methods). With this analysis, a similar tendency was seen. For example, DTG IgVH CDR3 had on average 3.4 hits per IgVH CDR3 sequence to herpes viral proteins in the category of sequences with 7 aa matches and 3 mismatches $(10-7 \mathrm{~m} / 3 \mathrm{~mm})$. The corresponding analysis on sequences from $B A L B / c$ mice $(10-7 \mathrm{~m} / 3 \mathrm{~mm})$ revealed 0.09 hits per CDR3 sequence (Figure 1G). L2-TG sequences had no hits in the categories shown.

In addition to the analysis above, 10 DTG anti-dsDNA IgVH CDR3 sequences were blasted toward all proteome sequences (i.e. an unrestricted, non-redundant protein sequence Blastp analysis). Results were manually screened for examples of hits toward non-bacterial pathogens (e.g. protozoa, fungi). Examples of the matches between one particular DTG anti-dsDNA IgVH CDR3 sequence and microbial proteomes can be seen in Figure 1H. This figure also includes examples of hits in the proteomes of two eukaryotic pathogens, the Leishmania major protozoa $(12-10 \mathrm{~m} / 2 \mathrm{~mm})$; and the Paracoccidioides brasiliensis fungus $(10-10 \mathrm{~m} / 0 \mathrm{~mm})$ - these latter results as obtained by Blastp, and manual screening.

\section{CDR3 Sequences from Neonatal L2-TG Mice have Reduced Matches with Microbial Proteomes}

The analyses above were based on sequences from adult mice, including sequences with mutations (see Figure 1D). To analyze the impact of antigenic selection of the $\mathrm{IgVH}$ repertoire, we used a 
data set from neonatal L2-TG mice [(35) see Materials and Methods] and repeated the microbial Blastp analysis. Although the IgVH CDR3 sequences from neonatal L2-TG mice demonstrated hits (i.e. exact matches, Figures 2A-C), this was nevertheless at a lower frequency than the IgVH CDR3 from DTG lupus mice (nine aa, $10 \%$ of that found in DTG; eight aa, 20\%; seven aa, $50 \%$ ), demonstrating an impact of mutation and diversification on identification of microbial mimics.

\section{Identification of Matches Between Anti-DNA CDR3 and Self Proteins}

In the next analysis, we compared the IgVH CDR3 sequences from DTG lupus mice with the mouse proteome, see Materials and Methods. We found IgVH CDR3 with five, six, seven matches as well as examples of hits with mismatches (Figures $\mathbf{3 A - C}$ ). As above, the mutated DTG sequences had higher frequency of matches. The frequencies of matches were 3-30× lower than those found toward microbial sequences (ratio of hits in mouse genome/hits in microbial genome. DTG: $28 \%$ of hits in the category seven aa $/ 0 \mathrm{~mm}, 3 \%$ in eight aa $/ 0 \mathrm{~mm}$; corresponding results in L2-TG: $12 \%$; 38\%) as shown in Figures 3C and 1E.

The IgVH CDR3 sequences in the DTG data set are enriched for sequences that have anti-dsDNA specificity with a preponderance of positively charged arginines (R) that mediate binding to DNA. Perhaps not surprisingly, many of the hits in the mouse genome were DNA/RNA-associated proteins with matching positively charged residues (Figure 3D). To generalize and investigate which $\operatorname{IgVH}$ CDR3 aa were more likely to be associated with hits toward the mouse proteome, we analyzed the BALB/c data set. This includes $>2000$ sequences that have been downloaded from NCBI. These sequences include specificities toward diverse antigens and are more representative for global $\mathrm{B}$ cell responses and unbiased IgVH CDR3. With this data set we found that the IgVH CDR3 residues $\mathrm{K}, \mathrm{D}, \mathrm{E}$ and $\mathrm{G}, \mathrm{Y}, \mathrm{L}$ and multiples thereof (e.g. more than $1 \mathrm{~K}$ per IgVH CDR3 sequence) were positively associated with hits (Table S1 in Supplementary Material; see Materials and Methods for algorithm). IgVH CDR3 R was however only increased in hits if it was found more than $4 \times$ (i.e., CAR. R. R. R), when it was three times as likely to be found in the matched sequences. These results suggest that arginines per se (as overrepresented in the DTG data set) were not directly associated with enriched hits in the mouse genome.

\section{Similarities of IgVH CDR3 Sequences with Histone Sequences}

The above analyses suggest that Th responses toward conventional autoantigen and Id may in fact be related in lupus. It has been suggested that histone-derived peptides constitute antigens for pathogenic Th cells in lupus (13). We therefore restricted the analysis to investigate IgVH CDR3 Id as potential mimics of histone sequences. Utilizing the sequences from the DTG mice with Iddriven lupus, we found IgVH CDR3 sequences with similarities to a histone $\mathrm{H} 2 \mathrm{~A}$ as well as $\mathrm{H} 4$, sequences previously identified as a Th cell antigens in lupus (13), Figure 4A. Similar matches were found for human IgVH CDR3 sequences when compared with human histone H2A and H4 (Figure S1 in Supplementary Material).

The above analyses searched for similarities between $\operatorname{IgVH}$ CDR3 sequences and genomic sequences, including histones. Reversing the analysis, we investigated if stretches of histone

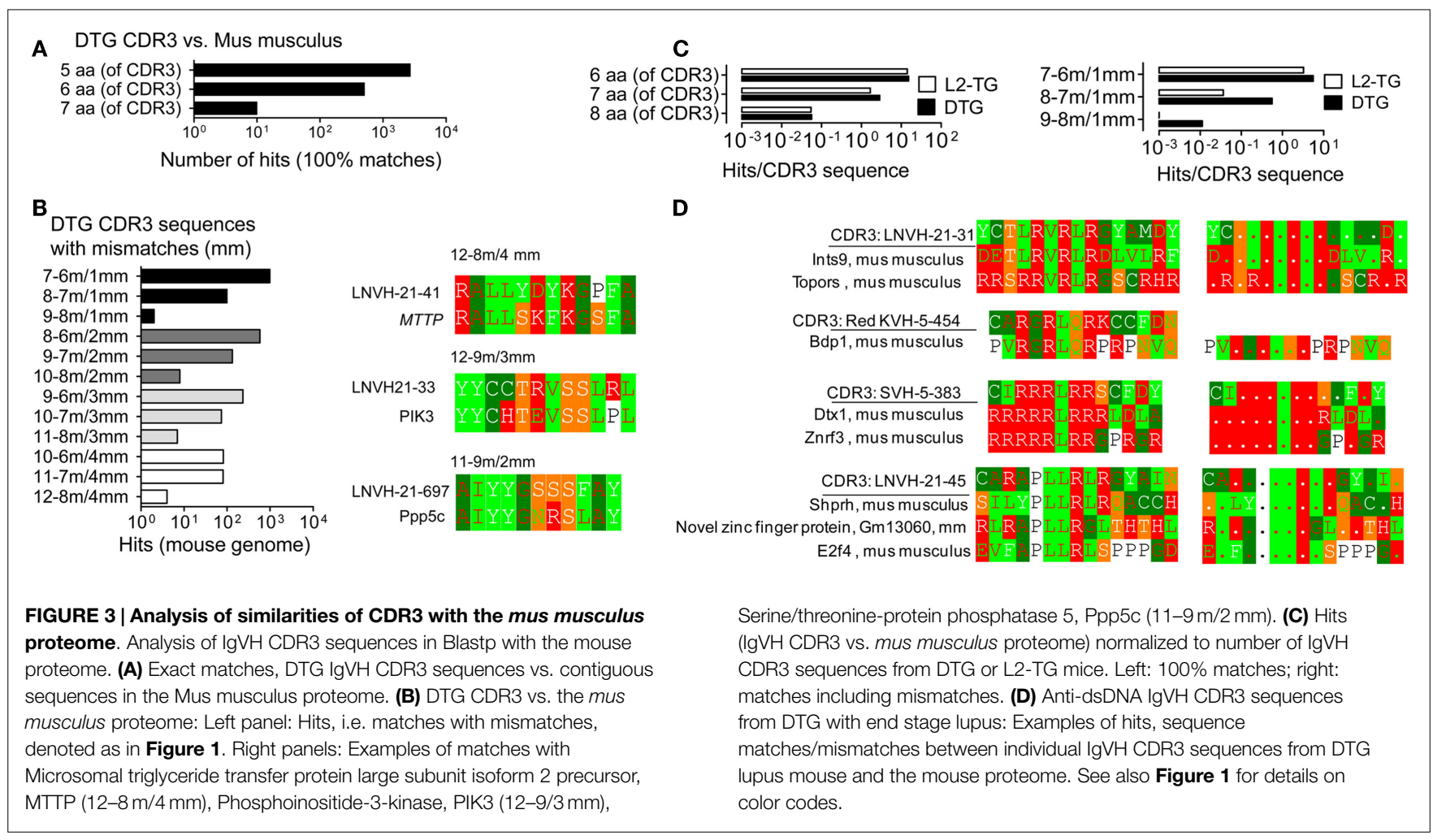




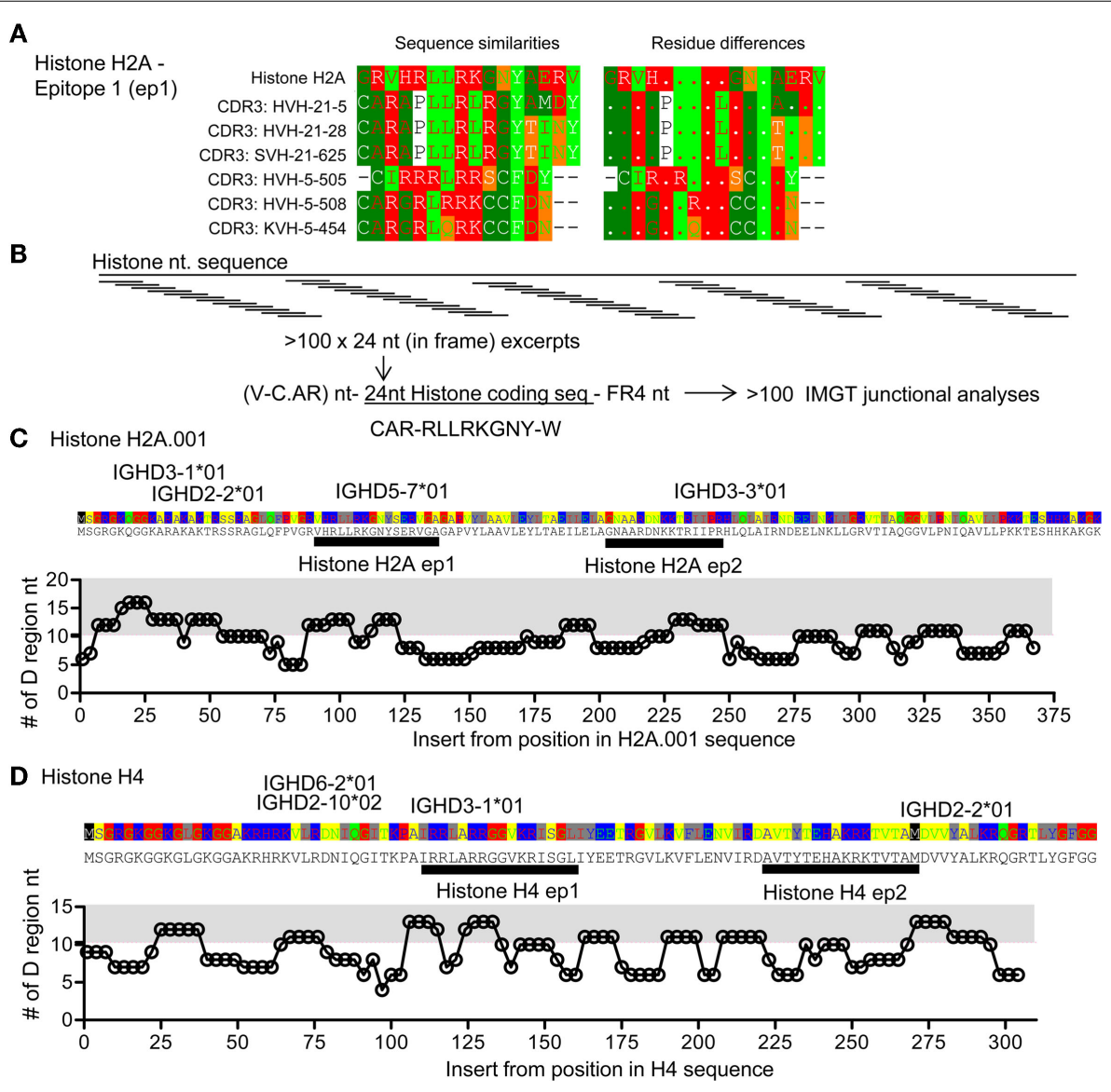

FIGURE 4 | Comparison of CDR3 and histone sequences; analysis of histone $\mathrm{H} 2 \mathrm{~A}$ sequence segments as part of $\mathrm{V}-\mathrm{D}-\mathrm{J}$ junctions.

(A) Examples of sequence comparisons of IgVH CDR3 sequences from DTG mice with end stage lupus and histone H2A, this epitope is denoted epitope 1 H2A-ep1. See also Figure S1 in Supplementary Material. (B) Artificial hybrid sequence files were made by inserting histone sequences - replacing the IgVH CDR3 to form model sequences. IgVH CDR3 regions are usually less than eight amino acids long, corresponding to 24 coding nucleotides. In frame $\mathrm{H} 2 \mathrm{~A}$ sequences were electronically generated i.e. HisH2A nt 1-24, nt 4-27, . ., nt 367-391 and electronically engrafted onto a random IgVH-segment. A random
FR4 was grafted onto this artificial lgVH - "Histone H2A-CDR3." Resulting model sequence files were up-loaded for IgVH junctional analysis at IMGT. (C) The lengths of suggested IMGT D-segments are plotted. The average D segment usage in BALB/c mice is $10.2 \mathrm{nt}$. Gray area: $>11 \mathrm{nt}$. HisH2A sequence is provided above the plot, possible $D$ gene segments are positioned according to highest levels of matching. Two epitopes are marked, histone H2A-ep 1 [see also (A)] and histone H2A-ep2. See also Figure S1 in Supplementary Material. (D) Analysis of histone $\mathrm{H} 4$ corresponding to that performed in (C). Two epitopes are marked histone $\mathrm{H} 4-\mathrm{ep} 1$ and histone $\mathrm{H} 4-\mathrm{ep} 2$, see also Figure $\mathbf{5}$ and Figure S1 in Supplementary Material. sequences could be coded by sequences similar to VDJ sequences, i.e., tested how well histone sequence excerpts could be accommodated within constraints of the VDJ junction. Focusing on histone $\mathrm{H} 2 \mathrm{~A}$ and histone $\mathrm{H} 4$, we pasted histone nt sequences onto a model IgVH, creating Vregion - histone segment-FR4 sequences. Resulting sequence files were uploaded into IMGT and subjected to VDJ junction analysis (Figure 4B; see Materials and Methods). With the histone $\mathrm{H} 2 \mathrm{~A}$, we found that histone inserts could be coded by $\mathrm{D}$ genes at three sites, Figure 4B. Using such excerpts, the D region segment lengths were above average ( $10.3 \mathrm{nt}$, from analysis of $>2000 \mathrm{BALB} / \mathrm{c}$ sequences, data not shown), Figure 4C. One of these epitopes corresponds to matches found with those described above (Figure 4A) called histone H2A epitope 1. Similarly, we identified four stretches of histone $\mathrm{H} 4$ that could be coded by other distinct $\mathrm{D}$ regions. One of these corresponded to the match seen in Figure 4A, histone H4, epitope 1.

\section{Anti-Histone Th Cell Responses in DTG Mice}

Following up the analyses above, we investigated if the lupus in DTG mice was associated with loss of tolerance toward histone motifs. In DTG mice with high ANA, but not in ANAlow DTG, we found significant responses toward both the H2A.001 and increased, but not significant response toward histone $\mathrm{H} 4$ epitopes (Figure 5). We proceeded to make Th cell lines towards each of these peptides. These were readily generated from DTG mice, but required more than five to six restimulations when Th cells were derived from single L2-TG or BALB/c mice (Figure 6A). The histone His4 ep1 (IRRLARRGGVKRISGL) has similarities with the IgVH CDR3 from the anti-DNA hybridoma 5.3 (CIRRRLRRSCFDYWG) but is dissimilar to IgVH CDR3 from the anti-DNA 21.5 (CARAPLLRLRGYAMDY). Strikingly, when purified $\mathrm{mAb}$ was used from the former, but not the latter, Th cell responses were elicited 


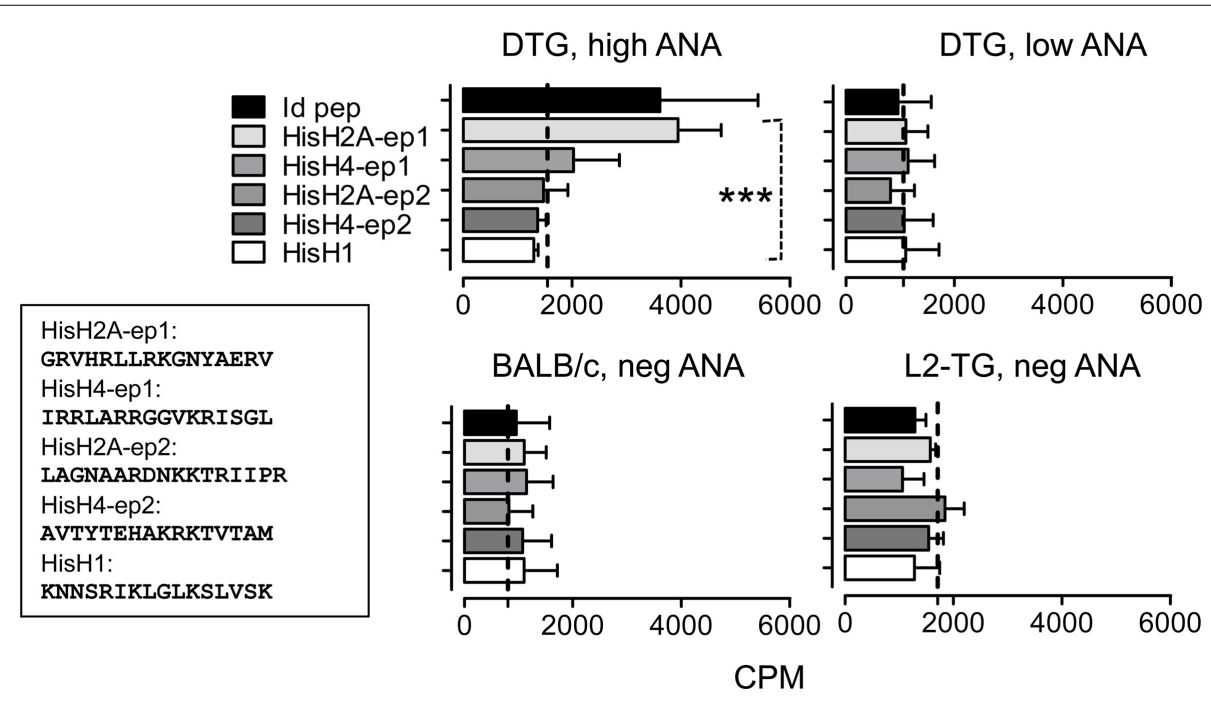

FIGURE 5 | Analysis of histone-specific Th cell responses in DTG mice and controls. Based on the predictions in Figure $\mathbf{4}$ and Figure S1 in Supplementary Material, histone peptide stretches with similarities with mouse IgVH CDR3 sequences were tested in DTG lupus mice. Lymph node Th cells were from controls (bottom) or high and low serum ANA DTG mice were tested for responses toward ld-peptide, or histone peptides from
$\mathrm{HisH} 1$, HisH2A, or HisH4 (see Materials and Methods and Figure 4 where $\mathrm{H}$ is $\mathrm{H} 2 \mathrm{~A}$ and $\mathrm{H} 4$ peptides are shown) presented by irradiated BALB/C splenocytes. Dotted line: Control without peptide, $n=6$. Upper left histograms (DTG, high ANA): One-way Anova, $p<0.0008$, with Tukey's Multiple Comparison test, $p<0.05$ for His2A-ep1 vs. other histone peptides and control).

\section{A}

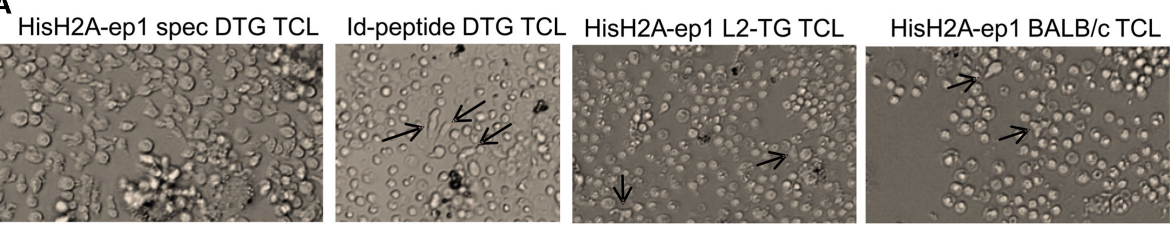

B

His4-ep1-specific DTG TCL His4-ep1-specific BALB TCL
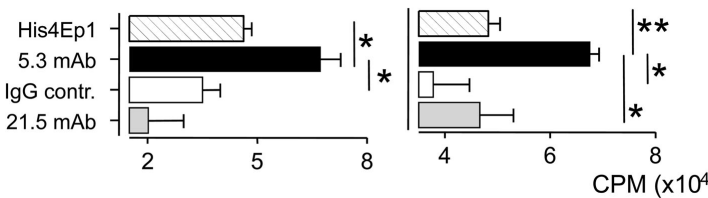

His2A-ep1specific L2-TG TCL

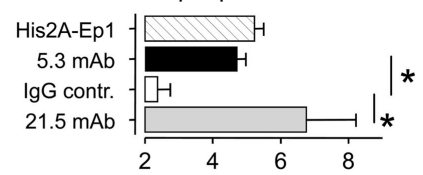

FIGURE 6 | Histone-specific T cell lines cross react to mAbs with CDR3 mimotopes. Th cell lines were cultured with irradiated APC and peptides in 10-day cycles, see Materials and Methods. (A) Micrographs Th cell lines (TCL): Left second cycle Th cells from DTG mice responding to HisH2A-ep1 peptide, Th cell blasts and proliferation clusters are seen. Middle left: third cycle Th cells from DTG mice responding to Id-peptide, only few Th cells are seen (arrows) admixed with APC. Middle, right: third cycle His2A-ep1 TCL Th cell line from
L2-TG mice, sparse Th cells (arrows) are indicated. Right: third cycle His2A-ep1 TCL from BALB/c. (B) Analysis of peptide specific TCL, cross reaction to mAbs: TCL lines from DTG, BALB/C and L2-TG were tested for responses toward $\mathrm{mAbs}$ with IgVH CDR3 sequence similarity with histone peptides. 5.3 mAb: IgVH CDR3 similar to His4Ep1. 21.5: IgVH CDR3 similar to His2A-ep1.

Polyclonal mouse IgG was control. Shown is proliferative response toward appropriate peptide or mAbs ( $n=4$, Student's $t$-test). in Th cell lines specific for the $\mathrm{H} 4$ peptide (Figure 6B, and data not shown). Reciprocally, Th cells specific for H2A peptide (GRVHRLLRKGNYAERV) responded to the $21.5 \mathrm{mAb}$ with CDR3 (CARAPLLRLRGYAMDY) and less to the $5.3 \mathrm{mAb}$, Figure 6B, and data not shown. The Th cell lines did not respond to Id-peptide from the transgene, data not shown. The anti-dsDNA mAbs were negative for $\mathrm{Id}^{+} \lambda 2^{315} \mathrm{~L}$ chains both in ELISA (data not shown) and by MALDI TOF mass spectrometry analysis (33).

\section{Discussion}

We found that IgVH CDR3 from the mutated repertoire of lupus mice had a surprisingly high rate of matches toward the microbial proteomes, eukaryotic pathogens (fungi and protozoa), as well as self-proteins. Results suggested that these antigenic determinants may potentially stimulate common Th cell populations - and that these disparate peptide sources could constitute a network of peptide mimics. The frequency of matches was increased in the 
data set derived from the DTG mice with lupus, compatible with mutation and diversification of the autoimmune repertoire. Moreover, even with a limited set of IgVH CDR3 regions from mice with lupus (176 sequences), it was possible to identify exact matches from microbial proteomes of up to nine contiguous aa. We also found examples in categories such as 11 aa including 10 matched aa and 1 mismatch; or longer stretches of for example 15 mers with 11 matches. Lupus mice with high levels of anti-dsDNA autoantibodies also had detectable Th cell responses toward histone determinants. Histone-reactive Th cell lines from such mice also responded with proliferation to stimulation when provided with anti-dsDNA mAbs with IgVH CDR3s that were similar to the histone epitopes, but did not respond to anti-dsDNA mAbs that had dissimilar CDR3s. Molecular mimics have been suggested in EAE (mice) and MS (humans), for example peptide mimics from pathogens were found to stimulate myelin basic protein (MBP)-specific Th cells $(1,2)$. However, these peptide epitope mimics had seven or five matched aa respectively across 15 mer stretches $(1,2)$. The current findings of up to 11 matched aa in 15 mers, and identity across 9mers suggest that idiotypes can provide a diverse pool of peptide mimics that may impact Th cell immunity.

The current results do not include analyses and prediction of peptide binding to MHC class II. TCR recognize peptides in the context of MHC, peptide mimicry could only occur if peptide sets could be presented to Th cells. In this regard, recent mathematical modeling and analysis of unbiased $\mathrm{IgVH}$ sequences from humans have revealed that $\mathrm{V}$ region peptides are especially well suited at binding MHC class II (37). The analysis demonstrated that the $\mathrm{CDR}$ regions had a unique propensity for MHC class II binding, and represent a frequent source of potential TCR exposed motifs (Th cell epitopes), (37).

It has previously been suggested that $\mathrm{V}$ region idiotypes may regulate immune responses, regulate clonal size, and also provide tonic signals for Th cells $(15,16,18-22,24,25,27-33$, $37,38)$. In the current setting, $\operatorname{IgVH}$ sequences were derived from lupus mice that developed a skewed oligoclonal repertoire enriched for cationic aa and especially arginines (33), which are aa that can mediate binding to dsDNA. These sequences were very similar to those found in other mouse models of lupus (33). We have previously demonstrated that Id-specific Th cells can induce anti-Histone autoantibodies (28) as well as anti-dsDNA antibodies, vasculitis, and nephritis $(30,31)$ and that both Th cells and B cells were necessary for development of the observed pathogenesis (30).

Lupus is associated with oligoclonal expansions of anti-dsDNA B cells $(9,11,33,39)$. B cells that are stimulated through the BCR in the absence of T cell help develop into anergic cells and undergo apoptosis, as reviewed in Ref. (40). Antigen specific Th cells can negate anergy and support conventional immune responses including germinal center reactions, development of plasma cells and autoantibody secretion $(39,41-43)$. Hence, anti-dsDNA B cell responses are dependent on help from Th cells, and both Th2 cells and follicular helper T cells have been directly linked to SLE $(9,11,39)$.

In this setting, B cells will present a skewed repertoire of IgVH CDR3 peptides on MHC class II molecules to Th cells. This as $\mathrm{B}$ cells process and present endogenous $\mathrm{BCR} \mathrm{V}$ regions on MHC class II to Th cells (18-20, 22, 25, 27-31, 33, 38). Idiotypes can also be presented by other antigen presenting cells, such as dendritic cells after receptor mediated endocytosis, and antigen processing $(15,16,20-22,24,25,28,32,38)$. In the case of lupus, uptake of immune complexes of dsDNA/antidsDNA could recruit Th responses directed against $\mathrm{V}$ region peptides. Recruitment would be facilitated by efficient antigen loading of immune complexes via Fc receptors, establishment of a pro-inflammatory microenvironment (serving as TLR ligands and inducing type I cytokines and chemokines) and contribute to a reduced regulatory activity, as has also been associated with Th cell responses directed toward antibody-based biologics (44).

The current results suggest that in settings with 1) skewed expression and presentation of endogenous $\mathrm{V}$ regions by B cells, 2) presentation of $\mathrm{V}$ regions from immune complexes by non-B cell APC, and 3) ongoing inflammation may lead to epitope spreading. Hence, it is possible that cationic CDR3 dsDNA-binding sequences may also provide mimics for Th cell responses directed against cationic peptides in dsDNA-associated proteins.

What is the potential for mimicry and TCR cross reactions? In general terms, TCR $\alpha \beta$ diversity has been estimated to potentially exceed $10^{20}$ as a result of nucleotide insertions and somatic recombination of gene segments (45). However, estimates suggest that there are $<10^{8}$ different antigen receptors in the naive $\mathrm{T}$ cell pool (46). In any case, these numbers are dwarfed by the potential number of antigenic peptide-MHC molecules related to pathogens. Nevertheless, the relatively small number of TCRs provides an effective immune recognition to most pathogens. To bridge the gap of many orders of magnitude, it was suggested that TCR recognition may be degenerate $(3,4,47)$. As a consequence, TCRs may be widely cross reactive. In fact, TCR cross reactivity has been implicated in both pathogenic and protective immunity in a number of diseases or infections (7, 48-52). TCR cross reactivity has recently been studied in context of class I (4) and class II (53). With regards to pMHC class I binding, the TCR $\alpha \beta$ was estimated to potentially bind $10^{6}$ peptides (4). This is more than three orders of magnitude higher than that found for MHC class II by pMHC display libraries in yeast (53). TCR specificity resides in the arrangement of the variable CDR3 loop that may interact with TCR contact residues of the peptides (53). Cross reactive peptides could harbor interchangeable aa similars (53), as has also described earlier $(5,7)$. In terms of the current results, it is likely that the cationic CDR3 peptides enriched for DNA-binding could provide a potential mimic for cationic DNA-associated peptide sequences.

As anti-dsDNA antibodies share canonical properties across mouse models as well as between species, Th cell responses toward such idiotypes may be accompanied with the potential for responses toward seemingly not related DNA-associated proteins. For example, histone H4 (aa 71-94) has been described to be a Th cell epitope in lupus mice and in SLE patients. This peptide contains the following DNA-associated motif: HAKRKTVTAMD (13). The sequence is comparable to the IgVH CDR3 from that of several previously described anti-DNA IgVH CDR3 derived 
peptides (33). If Th cells respond to cationic Id peptides of such $\mathrm{B}$ cells, it could be hypothesized that established Th cell responses would potentially cross react to peptide mimics from other DNAbinding proteins, including histones as well as bacterial sequences with the same flavor (14).

In addition to this mimicry on the level of peptide presentation, B cell immune responses toward pathogens may initiate antiDNA responses as demonstrated for pneumonia vaccines, and polysaccharide-binding antibodies $(54,55)$. In such situations expansion of anti-DNA B cells presenting a skewed CDR3 pMHC may result in Th responses that could cross react to a range of potential mimics. If so, the immune responses to these seemingly disparate peptides are related in Lupus.

\section{References}

1. Wucherpfennig KW. Mechanisms for the induction of autoimmunity by infectious agents. J Clin Invest (2001) 108:1097-104. doi:10.1172/JCI200114235

2. Kohm AP, Fuller KG, Miller SD. Mimicking the way to autoimmunity: an evolving theory of sequence and structural homology. Trends Microbiol (2003) 11:101-5. doi:10.1016/S0966-842X(03)00006-4

3. Wucherpfennig KW, Hafler DA, Strominger JL. Structure of human T-cell receptors specific for an immunodominant myelin basic protein peptide: positioning of T-cell receptors on HLA-DR2/peptide complexes. Proc Natl Acad Sci U S A (1995) 92:8896-900. doi:10.1073/pnas.92.19.8896

4. Wooldridge L, Ekeruche-Makinde J, Van Den Berg HA, Skowera A, Miles JJ, Tan MP, et al. A single autoimmune $\mathrm{T}$ cell receptor recognizes more than a million different peptides. J Biol Chem (2012) 287:1168-77. doi:10.1074/jbc. M111.289488

5. Wucherpfennig KW, Sette A, Southwood S, Oseroff C, Matsui M, Strominger JL, et al. Structural requirements for binding of an immunodominant myelin basic protein peptide to DR2 isotypes and for its recognition by human T cell clones. J Exp Med (1994) 179:279-90. doi:10.1084/jem.179.1.279

6. Quaratino S, Thorpe CJ, Travers PJ, Londei M. Similar antigenic surfaces, rather than sequence homology, dictate T-cell epitope molecular mimicry. Proc Natl Acad Sci U S A (1995) 92:10398-402. doi:10.1073/pnas.92.22.10398

7. Lang HL, Jacobsen H, Ikemizu S, Andersson C, Harlos K, Madsen L, et al. A functional and structural basis for TCR cross-reactivity in multiple sclerosis. Nat Immunol (2002) 3:940-3. doi:10.1038/ni835

8. Lipsky PE. Systemic lupus erythematosus: an autoimmune disease of B cell hyperactivity. Nat Immunol (2001) 2:764-6. doi:10.1038/ni0901-764

9. Vinuesa CG, Cook MC, Angelucci C, Athanasopoulos V, Rui L, Hill KM, et al. A RING-type ubiquitin ligase family member required to repress follicular helper T cells and autoimmunity. Nature (2005) 435:452-8. doi:10.1038/nature03555

10. Vinuesa CG, Sanz I, Cook MC. Dysregulation of germinal centres in autoimmune disease. Nat Rev Immunol (2009) 9:845-57. doi:10.1038/nri2637

11. Charles N, Hardwick D, Daugas E, Illei GG, Rivera J. Basophils and the T helper 2 environment can promote the development of lupus nephritis. Nat Med (2010) 16:701-7. doi:10.1038/nm.2159

12. Lewis JE, Fu SM, Gaskin F. Autoimmunity, end organ damage, and the origin of autoantibodies and autoreactive T cells in systemic lupus erythematosus. Discov Med (2013) 15:85-92.

13. Datta SK. Major peptide autoepitopes for nucleosome-centered $\mathrm{T}$ and $\mathrm{B}$ cell interaction in human and murine lupus. Ann N Y Acad Sci (2003) 987:79-90. doi:10.1111/j.1749-6632.2003.tb06035.x

14. Rekvig OP, Nossent JC. Anti-double-stranded DNA antibodies, nucleosomes, and systemic lupus erythematosus: a time for new paradigms? Arthritis Rheum (2003) 48:300-12. doi:10.1002/art.10739

15. Bogen B, Malissen B, Haas W. Idiotope-specific T cell clones that recognize syngeneic immunoglobulin fragments in the context of class II molecules. Eur J Immunol (1986) 16:1373-8. doi:10.1002/eji.1830161110

16. Hannestad K, Kristoffersen G, Briand JP. The T lymphocyte response to syngeneic lambda 2 light chain idiotopes. Significance of individual amino acids revealed by variant lambda 2 chains and idiotope-mimicking chemically synthesized peptides. Eur J Immunol (1986) 16:889-93. doi:10.1002/eji.1830160803

\section{Acknowledgments}

Ane Funderud, Inger Øynebråten, Peter O. Hofgaard, and Ole Audun Haabeth are thanked for critically reading the manuscript. The work was supported by The Research Council of Norway, FUGE, project number 17538; the University of Oslo; and Unifor (grants from Vivi Irene Hansen's, Gertrude \& Jack Nelson's, Signe \& Albert Bergsmarken's, and Henrik Homan's foundations).

\section{Supplementary Material}

The Supplementary Material for this article can be found online at http://journal.frontiersin.org/article/10.3389/fimmu.2015.00382

17. Mendlovic S, Shoenfeld Y, Bakimer R, Segal R, Dayan M, Mozes E. In vitro T-cell functions specific to an anti-DNA idiotype and serological markers in patients with systemic lupus erythematosus (SLE). J Clin Immunol (1988) 8:178-87. doi:10.1007/BF00917564

18. Weiss S, Bogen B. B-lymphoma cells process and present their endogenous immunoglobulin to major histocompatibility complex-restricted T cells. Proc Natl Acad Sci U S A (1989) 86:282-6. doi:10.1073/pnas.86.1.282

19. Weiss S, Bogen B. MHC class II-restricted presentation of intracellular antigen. Cell (1991) 64:767-76. doi:10.1016/0092-8674(91)90506-T

20. Bogen B, Dembic Z, Weiss S. Clonal deletion of specific thymocytes by an immunoglobulin idiotype. EMBO J (1993) 12:357-63.

21. Knupp CJ, Uner AH, Korthas C, Gavalchin J. Characterization of IdLNF1specific $\mathrm{T}$ cell clones from the (NZB x SWR)F1 murine model for systemic lupus erythematosus. Clin Immunol Immunopathol (1993) 68:273-82. doi:10. 1006/clin.1993.1128

22. Singh RR, Kumar V, Ebling FM, Southwood S, Sette A, Sercarz EE, et al. T cell determinants from autoantibodies to DNA can upregulate autoimmunity in murine systemic lupus erythematosus. J Exp Med (1995) 181:2017-27. doi:10. 1084/jem.181.6.2017

23. Williams WM, Staines NA, Muller S, Isenberg DA. Human T cell responses to autoantibody variable region peptides. Lupus (1995) 4:464-71. doi:10.1177/ 096120339500400608

24. Singh RR, Hahn BH. Reciprocal T-B determinant spreading develops spontaneously in murine lupus: implications for pathogenesis. Immunol Rev (1998) 164:201-8. doi:10.1111/j.1600-065X.1998.tb01221.x

25. Munthe LA, Kyte JA, Bogen B. Resting small B cells present endogenous immunoglobulin variable-region determinants to idiotope-specific CD4(+) $\mathrm{T}$ cells in vivo. Eur J Immunol (1999) 29:4043-52. doi:10.1002/(SICI) 1521-4141(199912)29:12<4043::AID-IMMU4043>3.0.CO;2-E

26. Dayan M, Segal R, Sthoeger Z, Waisman A, Brosh N, Elkayam O, et al. Immune response of SLE patients to peptides based on the complementarity determining regions of a pathogenic anti-DNA monoclonal antibody. J Clin Immunol (2000) 20:187-94. doi:10.1023/A:1006685413157

27. Snyder CM, Zhang X, Wysocki LJ. Negligible class II MHC presentation of B cell receptor-derived peptides by high density resting B cells. J Immunol (2002) 168:3865-73. doi:10.4049/jimmunol.168.8.3865

28. Munthe LA, Os A, Zangani M, Bogen B. MHC-restricted Ig V region-driven T-B lymphocyte collaboration: B cell receptor ligation facilitates switch to IgG production. J Immunol (2004) 172:7476-84. doi:10.4049/jimmunol.172.12. 7476

29. Snyder CM, Aviszus K, Heiser RA, Tonkin DR, Guth AM, Wysocki LJ. Activation and tolerance in CD4(+) T cells reactive to an immunoglobulin variable region. J Exp Med (2004) 200:1-11. doi:10.1084/jem.20031234

30. Munthe LA, Corthay A, Os A, Zangani M, Bogen B. Systemic autoimmune disease caused by autoreactive B cells that receive chronic help from Ig V regionspecific T cells. J Immunol (2005) 175:2391-400. doi:10.4049/jimmunol.175.4. 2391

31. Zangani M, Carlsen H, Kielland A, Os A, Hauglin H, Blomhoff R, et al. Tracking early autoimmune disease by bioluminescent imaging of NF-kappaB activation reveals pathology in multiple organ systems. Am J Pathol (2009) 174:1358-67. doi:10.2353/ajpath.2009.080700 
32. Feng F, Silvin CJ, Fiore NC, Stoll ML, Price KE, Shanley PS, et al. 17betaestradiol (E-2) administration to male (NZB x SWR)F(1) mice results in increased $\operatorname{Id}(\mathrm{LN}) \mathrm{F}(1)$-reactive memory T-lymphocytes and accelerated glomerulonephritis. Lupus (2012) 21:288-301. doi:10.1177/0961203311425519

33. Aas-Hanssen K, Funderud A, Thompson KM, Bogen B, Munthe LA. Idiotype-specific Th cells support oligoclonal expansion of anti-dsDNA B cells in mice with lupus. J Immunol (2014) 193:2691-8. doi:10.4049/jimmunol. 1400640

34. Kretschmer K, Jungebloud A, Stopkowicz J, Stoermann B, Hoffmann R, Weiss S. Antibody repertoire and gene expression profile: implications for different developmental and functional traits of splenic and peritoneal B-1 lymphocytes. J Immunol (2003) 171:1192-201. doi:10.4049/jimmunol.171.3.1192

35. Kretschmer K, Engel H, Weiss S. Strong antigenic selection shaping the immunoglobulin heavy chain repertoire of B-1a lymphocytes in lambda 2(315) transgenic mice. Eur J Immunol (2002) 32:2317-27. doi:10.1002/ 1521-4141(200208)32:8<2317::AID-IMMU2317>3.0.CO;2-0

36. Alamyar E, Giudicelli V, Li S, Duroux P, Lefranc MP. IMGT/HighV-QUEST: the IMGT(R) web portal for immunoglobulin (IG) or antibody and T cell receptor (TR) analysis from NGS high throughput and deep sequencing. Immunome Res (2012) 8:26. doi:10.1007/978-1-61779-842-9_32

37. Bremel RD, Homan EJ. Frequency patterns of T-cell exposed amino acid motifs in immunoglobulin heavy chain peptides presented by MHCs. Front Immunol (2014) 5:541. doi:10.3389/fimmu.2014.00541

38. Bogen B, Weiss S. Processing and presentation of idiotypes to MHC-restricted T cells. Int Rev Immunol (1993) 10:337-55. doi:10.3109/08830189309061709

39. Linterman MA, Rigby RJ, Wong RK, Yu D, Brink R, Cannons JL, et al. Follicular helper $\mathrm{T}$ cells are required for systemic autoimmunity. J Exp Med (2009) 206:561-76. doi:10.1084/jem.20081886

40. Cambier JC, Gauld SB, Merrell KT, Vilen BJ. B-cell anergy: from transgenic models to naturally occurring anergic B cells? Nat Rev Immunol (2007) 7:633-43. doi:10.1038/nri2133

41. Fulcher DA, Lyons AB, Korn SL, Cook MC, Koleda C, Parish C, et al. The fate of self-reactive $\mathrm{B}$ cells depends primarily on the degree of antigen receptor engagement and availability of T cell help. J Exp Med (1996) 183:2313-28. doi:10.1084/jem.183.5.2313

42. Cook MC, Basten A, Fazekas de St GB. Rescue of self-reactive B cells by provision of T cell help in vivo. Eur J Immunol (1998) 28:2549-58. doi:10.1002/ (SICI)1521-4141(199808)28:08<2549::AID-IMMU2549>3.0.CO;2-O

43. Seo SJ, Fields ML, Buckler JL, Reed AJ, Mandik-Nayak L, Nish SA, et al. The impact of $\mathrm{T}$ helper and $\mathrm{T}$ regulatory cells on the regulation of antidouble-stranded DNA B cells. Immunity (2002) 16:535-46. doi:10.1016/ S1074-7613(02)00298-4

44. van Schouwenburg PA, Rispens T, Wolbink GJ. Immunogenicity of anti-TNF biologic therapies for rheumatoid arthritis. Nat Rev Rheumatol (2013) 9:164-72. doi:10.1038/nrrheum.2013.4
45. Zarnitsyna VI, Evavold BD, Schoettle LN, Blattman JN, Antia R. Estimating the diversity, completeness, and cross-reactivity of the $\mathrm{T}$ cell repertoire. Front Immunol (2013) 4:485. doi:10.3389/fimmu.2013.00485

46. Arstila TP, Casrouge A, Baron V, Even J, Kanellopoulos J, Kourilsky P. A direct estimate of the human alphabeta T cell receptor diversity. Science (1999) 286:958-61. doi:10.1126/science.286.5441.958

47. Sewell AK. Why must T cells be cross-reactive? Nat Rev Immunol (2012) 12:669-77. doi:10.1038/nri3279

48. Wucherpfennig KW, Strominger JL. Molecular mimicry in T cell-mediated autoimmunity: viral peptides activate human $\mathrm{T}$ cell clones specific for myelin basic protein. Cell (1995) 80:695-705. doi:10.1016/0092-8674(95)90348-8

49. Benoist C, Mathis D. Autoimmunity provoked by infection: how good is the case for T cell epitope mimicry? Nat Immunol (2001) 2:797-801. doi:10.1038/ ni0901-797

50. Wucherpfennig KW. Polyspecificity of T cell and B cell receptor recognition. Semin Immunol (2007) 19:216-24. doi:10.1016/j.smim.2007.02.012

51. Welsh RM, Che JW, Brehm MA, Selin LK. Heterologous immunity between viruses. Immunol Rev (2010) 235:244-66. doi:10.1111/j.0105-2896. 2010.00897.x

52. Morris GP, Allen PM. How the TCR balances sensitivity and specificity for the recognition of self and pathogens. Nat Immunol (2012) 13:121-8. doi:10.1038/ ni. 2190

53. Birnbaum ME, Mendoza JL, Sethi DK, Dong S, Glanville J, Dobbins J, et al. Deconstructing the peptide-MHC specificity of T cell recognition. Cell (2014) 157:1073-87. doi:10.1016/j.cell.2014.03.047

54. el-Roiey A, Gross WL, Luedemann J, Isenberg DA, Shoenfeld Y. Preferential secretion of a common anti-DNA idiotype (16/6 Id) and anti-polynucleotide antibodies by normal mononuclear cells following stimulation with Klebsiella pneumoniae. Immunol Lett (1986) 12:313-9. doi:10.1016/0165-2478(86) 90036-2

55. Kowal C, Weinstein A, Diamond B. Molecular mimicry between bacterial and self antigen in a patient with systemic lupus erythematosus. Eur J Immunol (1999) 29:1901-11. doi:10.1002/(SICI)1521-4141(199906)29: 06<1901::AID-IMMU1901>3.0.CO;2-L

Conflict of Interest Statement: The authors declare that the research was conducted in the absence of any commercial or financial relationships that could be construed as a potential conflict of interest.

Copyright (c) 2015 Aas-Hanssen, Thompson, Bogen and Munthe. This is an openaccess article distributed under the terms of the Creative Commons Attribution License (CC BY). The use, distribution or reproduction in other forums is permitted, provided the original author(s) or licensor are credited and that the original publication in this journal is cited, in accordance with accepted academic practice. No use, distribution or reproduction is permitted which does not comply with these terms. 